\title{
Ion Suppression Study for Tetracyclines in Feed
}

\author{
Joaquim Chico, ${ }^{1}$ Frederique van Holthoon, ${ }^{2}$ and Tina Zuidema $^{2}$ \\ ${ }^{1}$ Departament de Química Analítica, Facultat de Química, Universitat de Barcelona, c/Martí i Franquès 1, 08028 Barcelona, Spain \\ ${ }^{2}$ RIKILT-Institute of Food Safety, Wageningen University and Research Center, Akkermaalsbos 2, 6708 WB, P.O. Box 230, 6700AE \\ Wageningen, The Netherlands \\ Correspondence should be addressed to Joaquim Chico, jchicorovira@gmail.com
}

Received 13 June 2012; Accepted 8 August 2012

Academic Editor: Andrew Cannavan

Copyright () 2012 Joaquim Chico et al. This is an open access article distributed under the Creative Commons Attribution License, which permits unrestricted use, distribution, and reproduction in any medium, provided the original work is properly cited.

Ion suppression in analysis of tetracyclines in feed was studied. The conventional analysis consists of a liquid extraction followed by a clean-up step using solid phase extraction (SPE) technique and analysis of the tetracyclines by liquid chromatography and mass spectrometric detection. Various strategies for extraction and cleanup were tested in the present work, and the effectiveness to decrease the ion suppression on the MS/MS signals was evaluated. Four sample treatment methods were tested with five different feed samples. Extraction solvents tested were Mcllvaine buffer and a mixture of McIlvaine buffer dichloromethane (3:1). SPE cartridges for cleanup were Oasis HLB, Oasis MCX, and Oasis MAX. The effectiveness of the methods was evaluated in terms of decreasing the ion suppression effect but also of decreasing the variability of ion suppression between samples. The method that provided the most satisfactory results involved a clean-up step based on SPE using mixed-mode cation exchange cartridges (Oasis MCX).

\section{Introduction}

Tetracyclines are a family of drugs belonging to the group of antibiotics. They are widely used in animal husbandry for therapeutic and prophylactic purposes. Oxytetracycline, tetracycline, chlortetracycline, and doxycycline are by far the most used antibiotics from this family. Their main chemical properties are their amphoteric behaviour due to their several acid-base equilibria and the tendency to act as chelating agents in presence of multivalent ions $[1,2]$. Figure 1 shows structures and $\mathrm{pKa}$ values of the tetracyclines studied. At $\mathrm{pH}$ values below 3 they are positively charged. At $\mathrm{pH}$ between $\mathrm{pKa} a_{1}$ and $\mathrm{pKa} a_{2}$ they are neutral (zwitterionic state), and above $\mathrm{pH} 8$ they are negatively charged.

The use of antibiotics in animal husbandry is strictly regulated to protect consumers, as the presence of antimicrobials residues in food products of animal origin can lead to resistance of bacteria to antibiotics. Therefore, the European Union has developed regulation concerning this issue $[3,4]$. Analysis and control of antibiotics in feedstuffs for animals has become an important issue as only authorized feedstuffs can be medicated under specific conditions as stated in Council Directive 90/167/EEC [5]. Use of tetracyclines as feed additives is forbidden in the EU since 2006, as stated in annex II of Commission Recommendation 2005/925/EC [6].

Feed contamination can occur depending on a large number of factors such as human error or handling procedures, but production practices have been identified as the main source [7].

Nowadays, liquid chromatography-tandem mass spectrometry (LC-MS/MS) is the technique of choice for the analysis of veterinary residues in food. The analysis of antibiotics in animal feed, though, has proved to be quite a challenge because of the high complexity and variability of the composition of the matrix. Numerous raw materials and additives are added into the feeds, including grains, seeds, beans, rice, and soy, and thus many interfering components, such as oils, fats, proteins, and salts can occur at very high levels. This complexity causes a strong effect of ion suppression. Ion suppression can be defined as a change in the efficiency of droplet formation or evaporation in the ion source of mass spectrometer, caused mainly by interfering matrix compounds. This affects the amount of charged analyte that reaches the detector and so the signal obtained for it. During the last years a growing concern on this issue has been reported [8-17]. Some factors such 


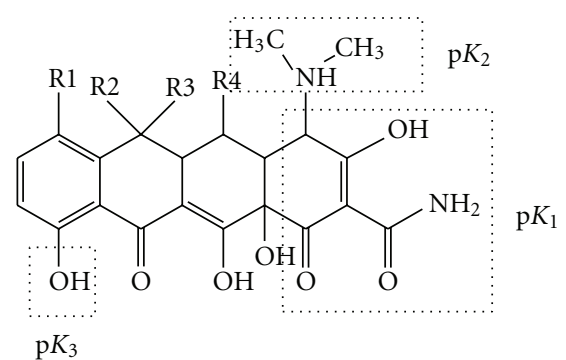

\begin{tabular}{llll}
\hline & $\mathrm{p} K_{1}$ & $\mathrm{p} K_{2}$ & $\mathrm{p} K_{3}$ \\
\hline OTC & 3.2 & 7.5 & 8.9 \\
TC & 3.3 & 7.8 & 9.6 \\
CTC & 3.3 & 7.6 & 9.3 \\
DC & 3 & 7.9 & 9.2 \\
\hline
\end{tabular}

FIGURE 1: Structures and $\mathrm{pKa}$ values of tetracyclines.

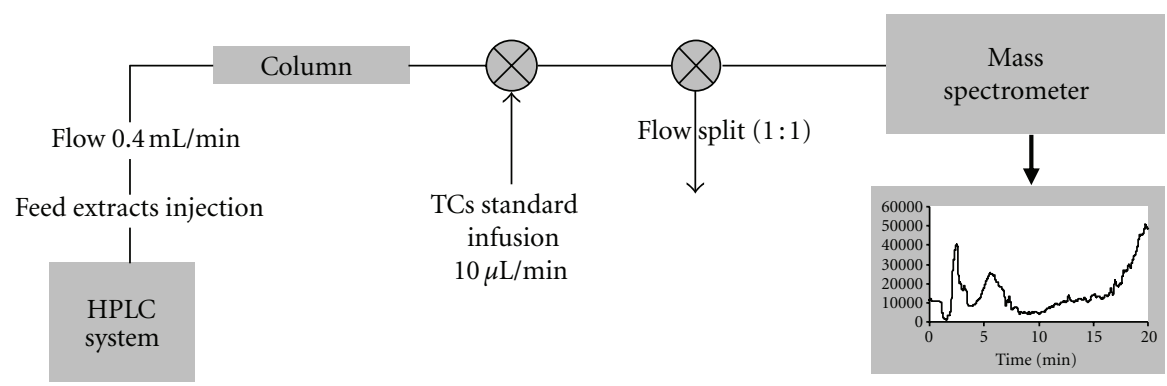

FIGURE 2: Schematic setup for ion suppression recording.

as mobile phase composition $[9,15]$ or the type of ion source and its geometry [17] have been reported to play a role in ion suppression, but matrix components reaching ion source are the most commonly reported of them. More knowledge on the removal of matrix interferences is needed to overcome ion suppression problems. Many authors have studied this phenomenon by improving sample treatment in residue analysis by HPLC-MS/MS in biological matrices such as whole blood, plasma, serum, or urine $[12-14,16,18]$. In the case of feed samples, it has been proved recently that they are an extreme case, regarding ion suppression, compared to other kind of matrices [19]. Moreover, the changeable composition of each individual feed leads to the obtention of sample extracts with high variation in matrix components, and that leads then to very different extents of ion suppression for each single feed sample. Therefore, not only is it much harder than with other kind of samples (like food) to avoid ion suppression effect, but it is also difficult to obtain at least a homogeneous sample-independent effect. This factor does not allow accurate quantification even when matrixmatched calibration approach is performed. A solution to overcome this effect has been found in the emergence of more isotopically labelled internal standards. The labelled internal standard coelutes with the analyte in question and has similar physicochemical properties. These internal standards, though, still do not ensure correct quantification in all cases [20]. Moreover, their commercial availability is still scarce, and they represent a high cost option. Dilution of the final extract to reduce matrix concentration is also a common option. However, when analyzing samples that may have been contaminated by error or by cross-contamination during production, levels can be very low (in the range of the few parts per billion) and so no great dilution factors are recommended. Presently, the only way to make completely sure that HPLC-MS/MS quantification is fully reliable is to apply standard addition calibration. This ensures a correct quantification of each individual feed. Unfortunately this quantification tool is very time consuming and cumbersome, resulting in only a few feeds being analysed per day, which is hardly affordable for laboratories which have to handle a high number of samples.

Development of analytical methodologies for analysis of veterinary drugs in feed by HPLC-MS/MS has started to increase in number for the last recent years. Although almost all authors are aware about ion suppression/enhancement phenomena in feed analysis, only a few of them have developed their methods including standard addition calibration $[21,22]$. Some others decided to perform this calibration technique by building calibration curve with spiked aliquots of the processed sample prior to HPLC-MS/MS analysis $[23,24]$, assuming that extraction recovery is a factor of much less impact in the final results than matrix effects. Others assume that their extraction and clean-up techniques are good enough to compensate this effect [25-27] or do not even mention it [28-30].

The aim of this work was to investigate some clean-up methodologies and to evaluate their effectiveness to reduce ion suppression in the analysis of antibiotic residues in feed by LC-MS. For this evaluation, some strategies mentioned in the literature $[9,11-13,18]$ were used. Tetracyclines were chosen as a model group to perform the experiments. No papers have been found reporting ion suppression concern in LC-MS/MS tetracycline analysis, and only one in LCMS analysis (single quadrupole and ion trap) in soils [31]. Several sample treatment procedures were tested and compared using different kinds of feed samples. 


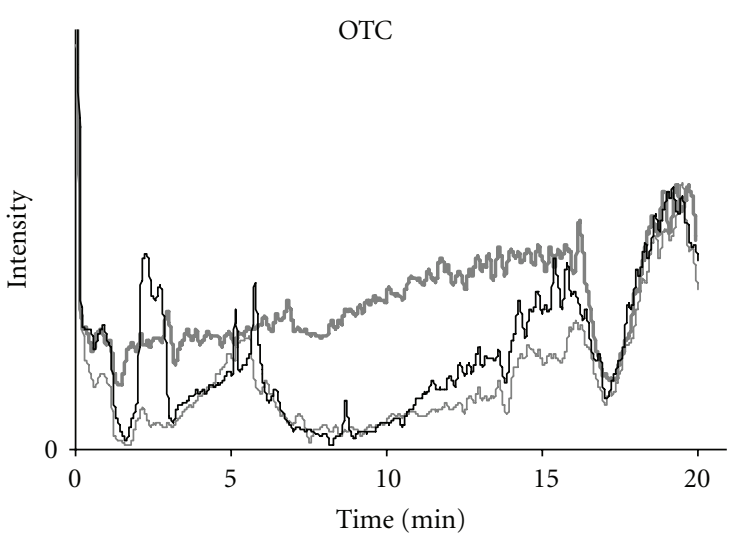

(a)

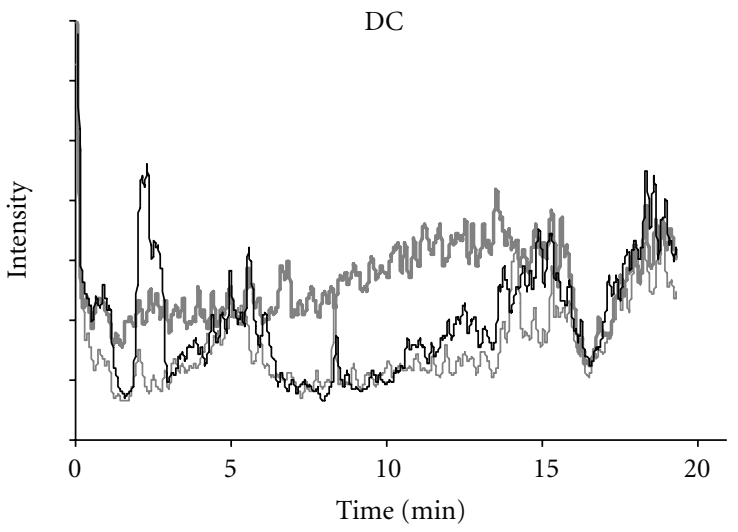

(c)

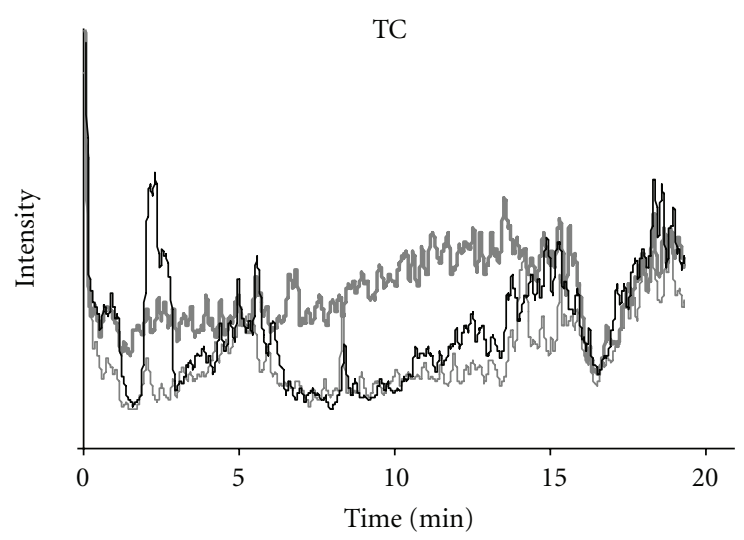

(b)

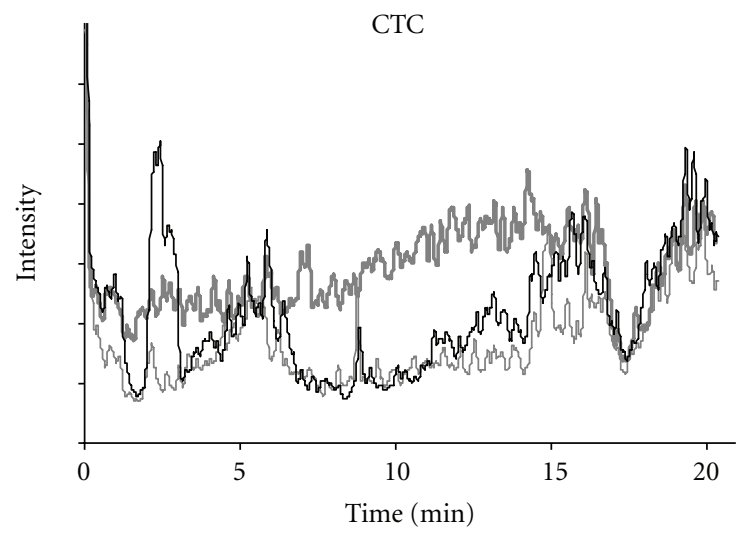

(d)

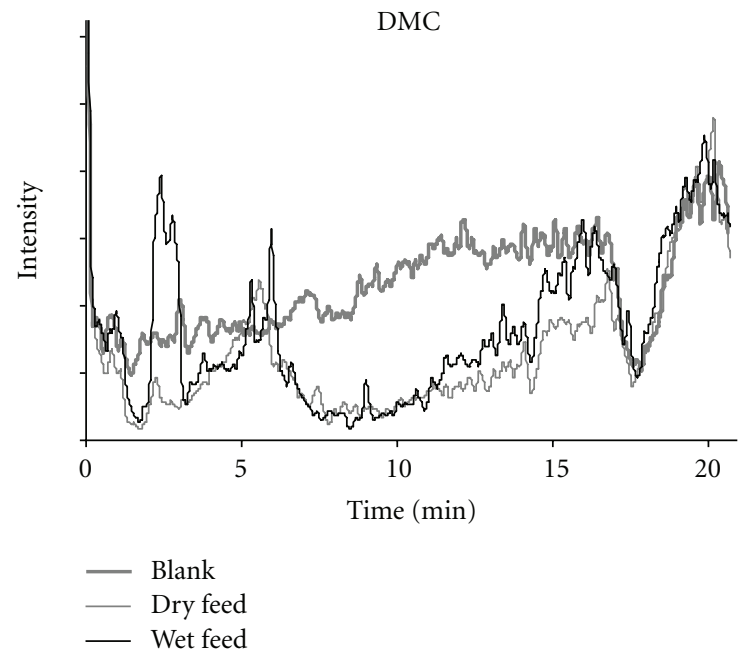

(e)

FIgURE 3: Ion suppression profiles of a blank, a dry, and a wet feed sample obtained with sample treatment method 1.

\section{Experimental}

2.1. Chemicals and Reagents. Oxytetracycline (OTC), tetracycline (TC), chlortetracycline (CTC), doxycycline (DC) and demeclocycline (DMC) were purchased from Sigma (St. Louis, MO, USA). Methanol $(\mathrm{MeOH})$ and acetonitrile (ACN) were obtained from Biosolve (Valkenswaard, The
Netherlands) and acetic acid (AA), ammonia solution 25\% $\mathrm{v} / \mathrm{v}\left(\mathrm{NH}_{3}\right)$, formic acid (FA), and dichloromethane (DCM) from Merck (Darmstadt, Germany). Solid reagents were purchased from Merck and included citric acid, potassium dihydrogen phosphate, ethylenediaminetetraacetic acid disodium salt (EDTA), and sodium hydroxide $(\mathrm{NaOH})$. All reagents were analytical-reagent grade. 
TABLE 1: Sample pretreatment methods tested for the cleanup of tetracyclines from animal feeds.

\begin{tabular}{|c|c|c|c|c|}
\hline & \multicolumn{4}{|c|}{ Method } \\
\hline & 1 & 2 & 3 & 4 \\
\hline Extraction & $\begin{array}{l}40 \mathrm{~mL} \text { McIlvaine } \\
\text { buffer-EDTA 0,1 M }\end{array}$ & $\begin{array}{l}40 \text { mL DCM McIlvaine } \\
\text { buffer-EDTA } 0,1 \mathrm{M} \\
(1: 3)\end{array}$ & $\begin{array}{l}40 \mathrm{~mL} \text { McIlvaine } \\
\text { buffer-EDTA 0,1 M }\end{array}$ & $\begin{array}{l}40 \mathrm{~mL} \text { McIlvaine } \\
\text { buffer-EDTA 0,1 M }\end{array}$ \\
\hline $\begin{array}{l}\text { SPE } \\
\text { Loading } \mathrm{pH} \\
\end{array}$ & 4.2 & 4.2 & 2.5 & 10 \\
\hline Cartridge & Oasis HLB (60 mg) & Oasis HLB (60 mg) & Oasis MCX (60 mg) & Oasis MAX (60 mg) \\
\hline \multirow{2}{*}{ Wash (2 mL) } & \multirow{2}{*}{$\mathrm{H}_{2} \mathrm{O}$} & \multirow{2}{*}{$\mathrm{H}_{2} \mathrm{O}$} & (1) FA $2 \%(v / v)$ & (1) $\mathrm{NH}_{3}$ \\
\hline & & & (2) $\mathrm{MeOH}$ & (2) $\mathrm{MeOH}$ \\
\hline Elution $(2 \mathrm{~mL})$ & $\mathrm{MeOH}$ & $\mathrm{MeOH}$ & $\mathrm{MeOH}: \mathrm{NH}_{3}(95: 5)$ & $\mathrm{MeOH}: \mathrm{FA}(95: 5)$ \\
\hline Final step & $\begin{array}{l}\text { Evaporation with } \mathrm{N}_{2} \\
\text { and reconstitution with } \\
\text { mobile phase }\end{array}$ & $\begin{array}{l}\text { Evaporation with } \mathrm{N}_{2} \\
\text { and reconstitution with } \\
\text { mobile phase }\end{array}$ & $\begin{array}{l}\text { Dilution with } 8 \mathrm{~mL} \\
\text { acetic acid } 10 \%(\mathrm{v} / \mathrm{v})\end{array}$ & $\begin{array}{l}\text { Dilution with } 8 \mathrm{~mL} \mathrm{H}_{2} \mathrm{O} \\
(\mathrm{v} / \mathrm{v})\end{array}$ \\
\hline
\end{tabular}

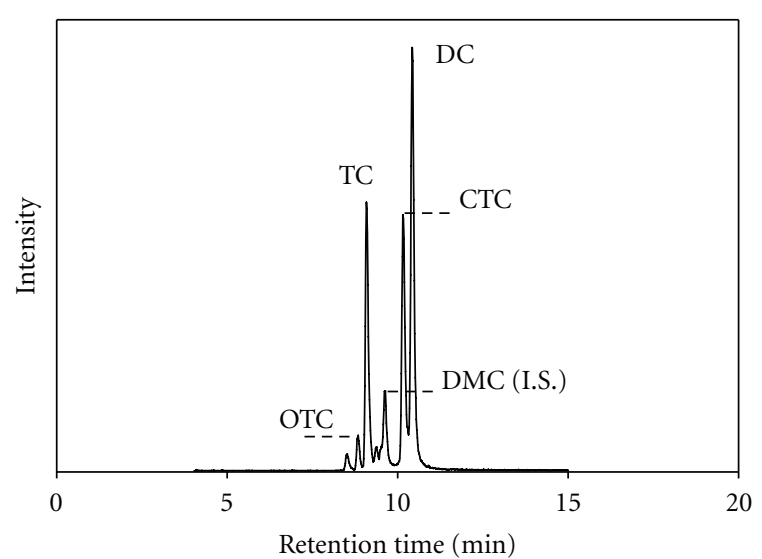

FIGURE 4: Chromatogram froms a standard injection of TC, CTC, OTC, DC, and DMC $\left(1 \mathrm{mg} \mathrm{L}^{-1}\right)$.

Standard solutions $\left(1000 \mathrm{mgL}^{-1}\right)$ were prepared in methanol monthly and stored at $4^{\circ} \mathrm{C}$. Mixtures of OTC, TC, CTC, and DC ( 10 and $100 \mathrm{mg} \mathrm{L}^{-1}$ ) were prepared by dilution of the concentrated solutions and stored at $4{ }^{\circ} \mathrm{C}$ for a week. An internal standard (IS) stock solution(DMC, $100 \mathrm{mg} \mathrm{L}^{-1}$ ) in $\mathrm{MeOH}$ was prepared monthly. Working standard solutions were prepared daily by mobile phase dilution of the 10 and $100 \mathrm{mg} \mathrm{L}^{-1}$ mixtures.

SPE materials were obtained from Waters (Micromass/Waters, Manchester, UK).

2.2. Sample Treatment Procedures. Five porcine feed samples were used for the study: one premix sample, two dry feed samples, and two slurry feed samples. Feeds were chosen that were representative for the range of different feeds available. Four different sample treatment procedures, which are summarized in Table 1, were tested. Sample weight was 2 grams, and extraction solution volume was $40 \mathrm{~mL}$. Extraction was carried out by means of a headover-head shaker for 20 minutes in all cases, and samples were subsequently centrifuged at $3000 \mathrm{rpm}$ for 15 minutes.
TABLE 2: LC-MS/MS precursor/product ion combinations (quantifier bold) monitored in MRM ESI positive mode.

\begin{tabular}{lccc}
\hline Tetracycline & $\begin{array}{c}\text { Retention } \\
\text { Time }(\mathrm{min})\end{array}$ & $\begin{array}{c}\text { Precursor ion } \\
(\mathrm{m} / \mathrm{z})\end{array}$ & $\begin{array}{c}\text { Product ions } \\
(\mathrm{m} / \mathrm{z})\end{array}$ \\
\hline TC & 9.2 & 445.2 & $\mathbf{4 1 0 . 1}, 154.1$ \\
CTC & 10.2 & 479.1 & $444.1, \mathbf{1 5 4 . 1}$ \\
DC & 10.4 & 445.2 & $\mathbf{4 2 8 . 1}, 154.1$ \\
OTC & 8.9 & 461.2 & $\mathbf{3 3 7 . 1}, 201.1$ \\
DMC & 9.7 & 465.1 & 154.1 \\
\hline
\end{tabular}

The $\mathrm{pH}$ of the extract was adjusted when necessary, and filtration through glass fiber filters was performed before SPE step. Final extracts obtained were filtered through $0.45 \mu \mathrm{m}$ nylon syringe filters before injection into the LC-MS/MS system.

2.3. LC-MS/MS Conditions. A Waters 2690 separations module HPLC system (Waters Corporation, USA) coupled to a Quattro Ultima tandem mass detector (Micromass/Waters, Manchester, UK), both operating under MassLynx software, was used for sample analysis. The mass spectrometer was operated in electrospray positive mode, and data acquisition was in multiple reaction monitoring mode (MRM). The precursor/product ions monitored are listed in Table 2. The source settings were as follows: capillary voltage $2.7 \mathrm{kV}$, source temperature $120^{\circ} \mathrm{C}$, desolvation temperature $300^{\circ} \mathrm{C}$, cone nitrogen gas flow $180 \mathrm{Lh}^{-1}$, and desolvation gas flow $580 \mathrm{Lh}^{-1}$. Argon $(3.2 \times 10-3 \mathrm{mbar})$ was used as the collision gas, and the multiplier was operated at $750 \mathrm{~V}$. The cone voltage was set at $20 \mathrm{~V}$, and collision energy changed during analysis depending on the analyte $(25 \mathrm{eV}$ for $\mathrm{TC}$ and DC, $26 \mathrm{eV}$ for OTC, and $30 \mathrm{eV}$ for CTC and DMC). The HPLC system was equipped with a Symmetry $\mathrm{C}_{18}(5 \mu \mathrm{m}$, $3.0 \times 150 \mathrm{~mm}$ column, Waters) at $10^{\circ} \mathrm{C}$. A binary gradient mobile phase was used at a flow rate of $0.4 \mathrm{~mL} \mathrm{~min}^{-1}$ with solvent A (ammonium acetate $1 \mathrm{mM}, \mathrm{pH} 2.6$ ) and solvent $\mathrm{B}$ (ammonium acetate $10 \mathrm{mM}: \mathrm{ACN}, 10: 90$ ). The gradient started isocratic for $1 \mathrm{~min}$ at $0 \% \mathrm{~B}$, followed by a linear 


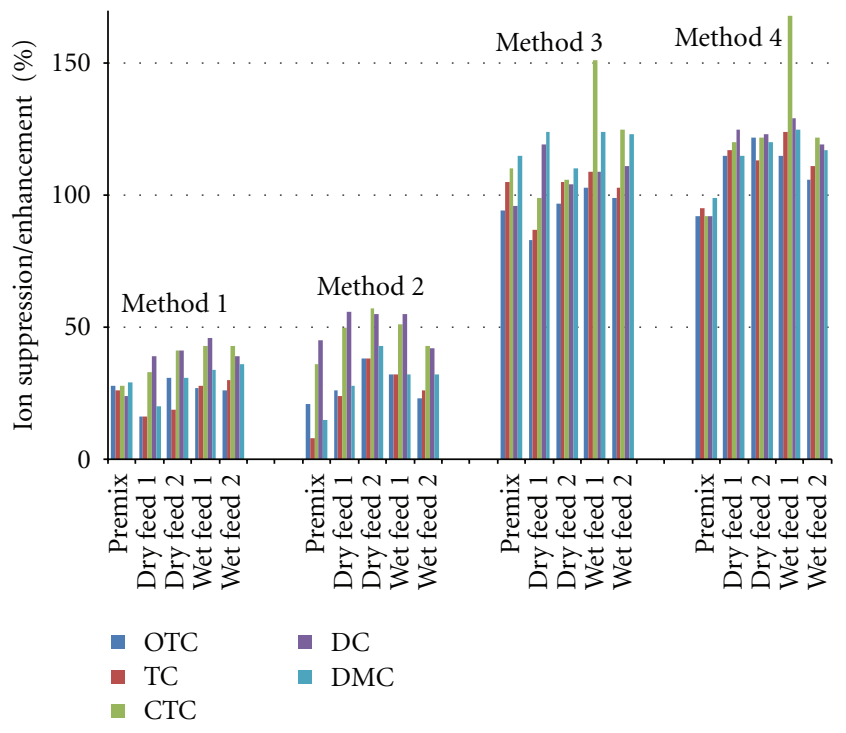

FIGURE 5: Quantitative results of ion suppression/enhancement for all analytes in all matrices and extraction methods tested.

increase to $50 \% \mathrm{~B}$ in $9 \mathrm{~min}$. The gradient remained isocratic at $50 \% \mathrm{~B}$ for $3 \mathrm{~min}$. Subsequently the gradient linearly increased to $100 \% \mathrm{~B}$ in $1 \mathrm{~min}$. The gradient remained at this $\% \mathrm{~B}$ for a further $3 \mathrm{~min}$. Afterwards the gradient returned to $0 \% \mathrm{~B}$ for equilibration of the column. Sample injection volume was $10 \mu \mathrm{L}$.

2.4. Qualitative Assessment of Ion Suppression. The experiments for qualitative assessment of ion suppression were carried out using a postcolumn infusion setup coupled to the chromatographic system described in Section 2.3 through a $\mathrm{T}$ piece. The setup is shown in Figure 2. The infusion pump flushes a constant flow at $10 \mu \mathrm{L} \mathrm{min}{ }^{-1}$ of a $5 \mathrm{mg} \mathrm{L}^{-1}$ standard solution of all tetracyclines in mobile phase. The quantification transition for each tetracycline is monitored in the MS/MS system. When mobile phase is injected into the system, a reference baseline for each transition is obtained due to the constant infusion of the standard solution of analytes. When feed extracts free from tetracyclines are injected, ion suppression profiles for each transition are obtained, and the influence of ion suppression in the tetracyclines infusion baseline due to the eluted matrix components can be evaluated. In fact, these profiles show the effect of compounds eluting from the chromatographic system on the analytes MS/MS signals. The signal intensity of the baseline decreases when matrix components causing ion suppression elute, and it increases when substances enhancing ionization elute.

Observing the signal variation at the time window where every analyte elutes, a good qualitative prediction can be made, whether suppression or enhancement are expected for that analyte.

2.5. Quantitative Assessment of Ion Suppression. $1 \mathrm{mg} \mathrm{L} \mathrm{L}^{-1}$ tetracyclines standard solutions and matrix-matched recovery standards (MMRSs) at the same concentration were injected into the LC-MS/MS system. MMRSs are extracts from blank feed samples that have been spiked with the analytes at the end of the sample treatment process. Ion suppression or enhancement percentages were determined for each tetracycline as the peak area ratio of the MMRS to the standard in solution multiplied by 100 . Values lower than $100 \%$ were an indicative of ion suppression whereas values higher than $100 \%$ indicated ion enhancement.

For each analyte, the response factors (Area analyte $_{\text {/Area }}$ IS in the 5 studied feed samples were determined in MMRS for the set of samples. Each MMRS was analysed by triplicate, and the average was calculated. The RSD (\%) between the averages of the five tested samples $(n=5)$ was used to quantify the variation in ion suppression due to differences between feed samples for each sample treatment method.

\section{Results and Discussion}

Four different sample treatments, summarized in Table 1, were tested in this study. All of them are based in an extraction step using McIlvaine buffer-EDTA $0.1 \mathrm{M}$ ( $\mathrm{pH}$ 4.2) and a further clean-up step of solid phase extraction. McIlvaine buffer has been extensively reported to be efficient for tetracycline extraction in a large number of matrices, as stated in some reviews $[1,2]$.

Method 1 is currently in use at this laboratory for routine analysis. In this method, the cleanup of the extracts is performed with the reversed phase Oasis HLB cartridges. The loading of the extract into the cartridge does not require any $\mathrm{pH}$ adjustment since the maximum interaction of TCs with the sorbent occurs when the neutral form of the analytes is prevalent, like at $\mathrm{pH} 4.2$ (Figure 1). Finally the elution of TCs is achieved with methanol.

The effect of addition of dichloromethane (DCM) in the extraction step was investigated in method 2, as DCM might assist to the removal of some nonpolar matrix compounds 


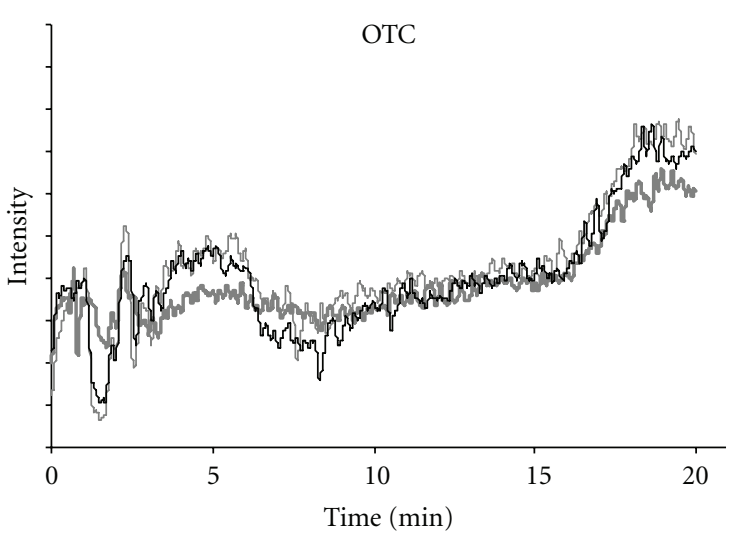

(a)

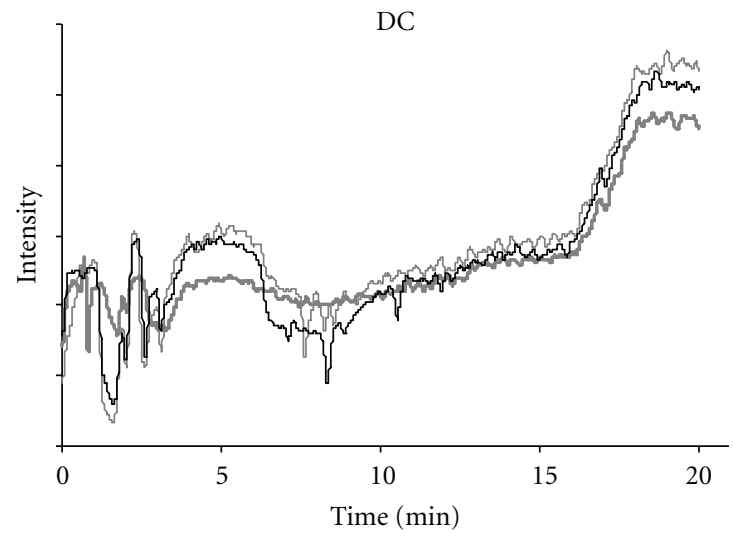

(c)

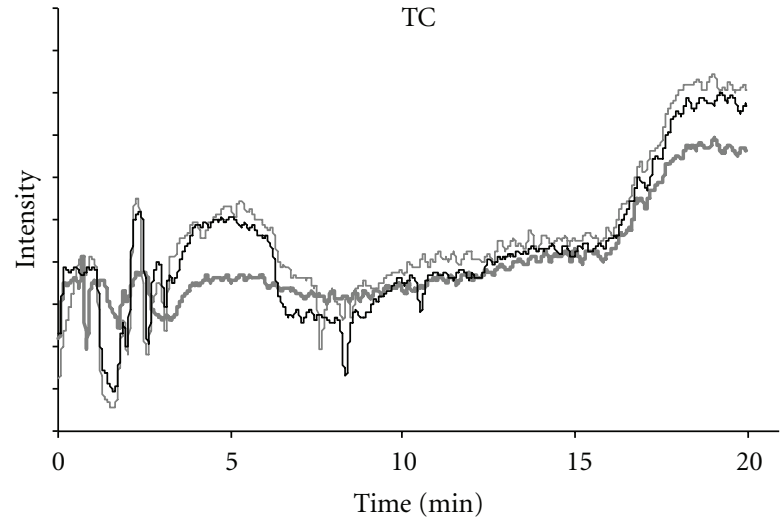

(b)

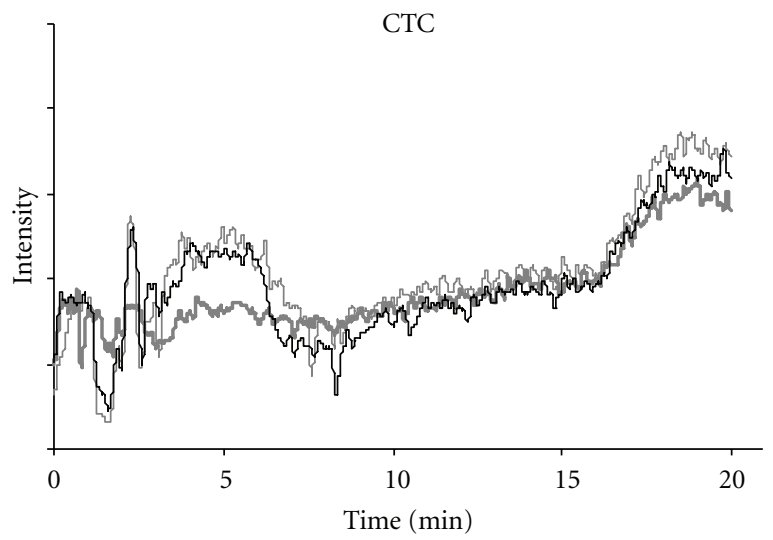

(d)

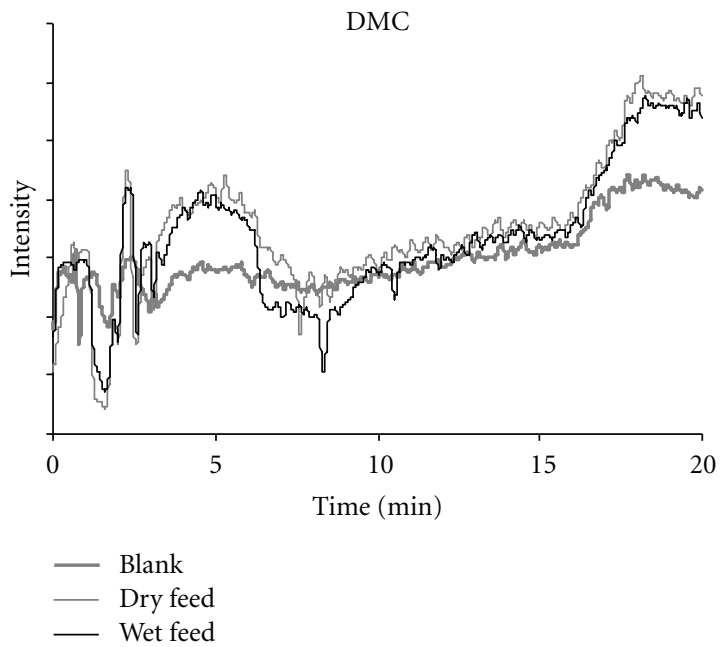

(e)

FIGURE 6: Ion suppression profiles of a blank, a dry, and a wet feed sample obtained with sample treatment method 3.

and thus provide cleaner extracts. After extraction, the aqueous layer was processed throughout an Oasis HLB cartridge as in method 1.

The $\mathrm{pH}$ of the Mcllvaine buffer extract was modified after extraction in methods 3 and 4 to reach a suitable $\mathrm{pH}$ for SPE. Mixed-mode cation exchange (Oasis MCX, method 3) or mixed-mode anion exchange (Oasis MAX, method 4) cartridges were used. These cartridges base their performance in a combination of ion exchange and reverse phase mechanisms. Therefore, they are expected to be more selective for targeted analytes and so to provide a more efficient cleanup [18, 32-34]. 
For method 3, before loading into the cartridge, $\mathrm{pH}$ of the extract was decreased to 2.5 in order to have the analytes positively charged. Theoretically SPE cartridge performance may be compromised, as the $\mathrm{pH}$ is only slightly lower than $\mathrm{pKa} a_{1}$ (Table 1). However decreasing $\mathrm{pH}$ more is not recommended, as it has been reported to induce epimerization of tetracyclines $[1,2]$. At these conditions good SPE recoveries for all analytes were obtained for the complete optimized method (74-100\%).

For method 4, $\mathrm{pH}$ of the extract was brought to 10 to ensure that all analytes were in anionic form. At these conditions, SPE recoveries were good for the analytes (97$100 \%$ ) except for CTC (ca. 30\%). This is possibly due to partial degradation of this analyte to iso-CTC, as this analyte is particularly prone to form this derivative at high $\mathrm{pH}$ values [2].

Ion suppression profiles of feed extracts obtained for the four sample pretreatment methods were recorded. These profiles were studied separately for each analyte in every feed extract and compared. Focusing in the behaviour of the profile at the retention time at which every tetracycline elutes, and comparing it with the reference "blank" signal (which corresponds to the injection of mobile phase), a good qualitative assessment can be made, whether suppression or enhancement are expected in a significant extent for each tetracycline in each studied feed extract.

Ion suppression profiles of a blank, a dry and a slurry (wet) feed sample extract obtained with sample treatment method 1 are shown in Figure 3. Both feeds exhibit ion suppression in the chromatogram time window where tetracyclines elute (8-12 min, Figure 4).

A similar trend was observed when using method 2. The addition of DCM to the extraction solution was therefore not an improvement. Numeric results from the ion suppression quantification experiment agreed with these results, and the suppression factors (see Section 2.5) for all the analytes in the set of feed samples were clearly below $100 \%$ for both methods (Figure 5).

Some improvements were clearly observed in the ion suppression profiles of the extracts obtained according to methods 3 and 4. No ion suppression or enhancement was observed in the profiles between 8 and $12 \mathrm{~min}$ As a matter of example Figure 6 shows the ion suppression profiles of a blank, a dry and a slurry (wet) feed sample extract obtained with method 3. Similar profiles were obtained when using method 4. The results of the ion suppression quantification experiment are consistent with these qualitative results. The percentage of signal obtained in $1 \mathrm{mgL}^{-1}$ MMRS in the five feeds studied compared to a standard solution was close to $100 \%$ (Figure 5). Only in the case of CTC in one of the two slurry feeds, significant enhancement $(>150 \%)$ was found, what seems to show that this sample contains some particular substances that enhance the ionization of this particular analyte under the mentioned sample treatment conditions. The overall clear improvement provided by methods 3 and 4 has been achieved by the combination of the use of ion exchange cartridges (more selective) and the dilution of the extracts instead of evaporation and reconstitution (less introduction of contaminants and human error).
TABLE 3: Variation (RSD \%) between the five different studied feed samples processed with the four different sample pretreatment methods at $1 \mathrm{mg} \mathrm{L}^{-1}$.

\begin{tabular}{lcccc}
\hline & \multicolumn{4}{c}{ Method } \\
& 1 & 2 & 3 & 4 \\
& RSD (\%) & RSD (\%) & RSD (\%) & RSD (\%) \\
\hline TC & 12.3 & 19.4 & 11.1 & 2.6 \\
CTC & 17.9 & 25.8 & 12.3 & 21.1 \\
DC & 30.8 & 39.7 & 4.9 & 6.5 \\
OTC & 14.7 & 24.3 & 9.4 & 8.6 \\
\hline
\end{tabular}

As expected, a high variation between the different feed samples was found for all tetracyclines (Table 3 ). Methods 3 and 4 provide the best results regarding the variability on the response factors (Area $a_{\text {analyte }} /$ Area $_{\text {IS }}$ ) in MMS due to the feed sample. CTC is an exception (method 4) due to degradation at $\mathrm{pH}$ 10. That indicates that the extracts obtained for the five feeds with these two methods are more uniform than the ones obtained by methods 1 and 2 .

\section{Conclusions}

Ion suppression in LC-MS/MS analysis of tetracyclines in feed was studied. The study of this phenomenon in feed samples has proven to be of a high level of complexity. Four sample pretreatment methods were tested with five different feed samples in terms of ion suppression profiles, ion suppression quantification, and variation. The method that seemed to provide less ion suppression and more uniform extracts without significant degradation of any analyte was method 3, which involved SPE with Oasis MCX cartridges. Although these results are still not sufficient to replace the current protocol (method 1) which relies heavily on standard addition, they provided valuable information for future research, which should include studies with larger numbers of different feed samples, different concentration levels, and different levels of extract dilution.

\section{References}

[1] H. Oka, Y. Ito, and H. Matsumoto, "Chromatographic analysis of tetracycline antibiotics in foods," Journal of Chromatography A, vol. 882, no. 1-2, pp. 109-133, 2000.

[2] C. R. Anderson, H. S. Rupp, and W. H. Wu, "Complexities in tetracycline analysis - chemistry, matrix extraction, cleanup, and liquid chromatography," Journal of Chromatography A, vol. 1075, no. 1-2, pp. 23-32, 2005.

[3] Commission Decision. Regulation (EC) No 882/2004 of the European Parliament and of the Council of 29 April 2004 on official controls performed to ensure the verification of compliance with feed and food law, animal health and animal welfare rules, 2004.

[4] Regulation. Regulation (EC) No 1831/2003 of the European Parliament and of the Council of 22 September 2003 on additives for use in animal nutrition, 2003.

[5] Council Directive 90/167/EEC. Council Directive 90/167/EEC of 26 March 1990 laying down the conditions governing the 
preparation, placing on the market and use of medicated feedingstuffs in the Community, 1990.

[6] Commision Recommendation 2005/925/EC of 14 December 2005 on the coordinated inspection programme in the field of animal nutrition for the year 2006 in accordance with Council Directive 95/53/EC.

[7] J. D. G. McEvoy, "Contamination of animal feedingstuffs as a cause of residues in food: a review of regulatory aspects, incidence and control," Analytica Chimica Acta, vol. 473, no. 1-2, pp. 3-26, 2002.

[8] L. L. Jessome and D. A. Volmer, "Ion suppression: a major concern in mass spectrometry," LC-GC North America, vol. 24, no. 5, pp. 498-510, 2006.

[9] T. M. Annesley, "Ion suppression in mass spectrometry," Clinical Chemistry, vol. 49, no. 7, pp. 1041-1044, 2003.

[10] J. P. Antignac, K. De Wasch, F. Monteau, H. De Brabander, F. Andre, and B. Le Bizec, "The ion suppression phenomenon in liquid chromatography-mass spectrometry and its consequences in the field of residue analysis," Analytica Chimica Acta, vol. 529, no. 1-2, pp. 129-136, 2005.

[11] Y. Hsieh, M. Chintala, H. Mei et al., "Quantitative screening and matrix effect studies of drug discovery compounds in monkey plasma using fastgradient liquid chromatography/tandem mass spectrometry," Rapid Communications in Mass Spectrometry, vol. 15, no. 24, pp. 2481-2487, 2001.

[12] B. K. Matuszewski, M. L. Constanzer, and C. M. Chavez-Eng, "Strategies for the assessment of matrix effect in quantitative bioanalytical methods based on HPLC-MS/MS," Analytical Chemistry, vol. 75, no. 13, pp. 3019-3030, 2003.

[13] C. Müller, P. Schäfer, M. Störtzel, S. Vogt, and W. Weinmann, "Ion suppression effects in liquid chromatographyelectrospray-ionisation transport-region collision induced dissociation mass spectrometry with different serum extraction methods for systematic toxicological analysis with mass spectra libraries," Journal of Chromatography B, vol. 773, no. 1, pp. 47-52, 2002.

[14] R. Bonfiglio, R. C. King, T. V. Olah, and K. Merkle, "The effects of sample preparation methods on the variability of the electrospray ionization response for model drug compounds," Rapid Communications in Mass Spectrometry, vol. 13, no. 12, pp. 1175-1185, 1999.

[15] C. R. Mallet, Z. Lu, and J. R. Mazzeo, "A study of ion suppression effects in electrospray ionization from mobile phase additives and solid-phase extracts," Rapid Communications in Mass Spectrometry, vol. 18, no. 1, pp. 49-58, 2004.

[16] J. X. Shen, R. J. Motyka, J. P. Roach, and R. N. Hayes, "Minimization of ion suppression in LC-MS/MS analysis through the application of strong cation exchange solidphase extraction (SCX-SPE)," Journal of Pharmaceutical and Biomedical Analysis, vol. 37, no. 2, pp. 359-367, 2005.

[17] M. Holčapek, K. Volná, P. Jandera et al., "Effects of ion-pairing reagents on the electrospray signal suppression of sulphonated dyes and intermediates," Journal of Mass Spectrometry, vol. 39, no. 1, pp. 43-50, 2004.

[18] E. Chambers, D. M. Wagrowski-Diehl, Z. Lu, and J. R. Mazzeo, "Systematic and comprehensive strategy for reducing matrix effects in LC/MS/MS analyses," Journal of Chromatography B, vol. 852, no. 1-2, pp. 22-34, 2007.

[19] H. G. J. Mol, P. Plaza-Bolaños, P. Zomer, T. C. De Rijk, A. A. M. Stolker, and P. P. J. Mulder, "Toward a generic extraction method for simultaneous determination of pesticides, mycotoxins, plant toxins, and veterinary drugs in feed and food matrixes," Analytical Chemistry, vol. 80, no. 24, pp. 9450-9459, 2008.
[20] N. Lindegardh, A. Annerberg, N. J. White, and N. P. J. Day, "Development and validation of a liquid chromatographictandem mass spectrometric method for determination of piperaquine in plasma. Stable isotope labeled internal standard does not always compensate for matrix effects," Journal of Chromatography B, vol. 862, no. 1-2, pp. 227-236, 2008.

[21] L. Kantiani, M. Farré, J. M. Grases I Freixiedas, and D. Barceló, "Determination of antibacterials in animal feed by pressurized liquid extraction followed by online purification and liquid chromatography- electrospray tandem mass spectrometry," Analytical and Bioanalytical Chemistry, vol. 398, no. 3, pp. 1195-1205, 2010.

[22] F. Van Holthoon, P. P. J. Mulder, E. O. Van Bennekom, H. Heskamp, T. Zuidema, and H. J. A. Van Rhijn, "Quantitative analysis of penicillins in porcine tissues, milk and animal feed using derivatisation with piperidine and stable isotope dilution liquid chromatography tandem mass spectrometry," Analytical and Bioanalytical Chemistry, vol. 396, no. 8, pp. 3027-3040, 2010.

[23] M. J. G. de la Huebra, U. Vincent, and C. von Holst, "Determination of semduramicin in poultry feed at authorized level by liquid chromatography single quadrupole mass spectrometry," Journal of Pharmaceutical and Biomedical Analysis, vol. 53, no. 4, pp. 860-868, 2010.

[24] U. Vincent, Z. Ezerskis, M. Chedin, and C. von Holst, "Determination of ionophore coccidiostats in feeding stuffs by liquid chromatography-tandem mass spectrometry. Part II. Application to cross-contamination levels and non-targeted feed," Journal of Pharmaceutical and Biomedical Analysis, vol. 54, no. 3, pp. 526-534, 2011.

[25] M. Cronly, P. Behan, B. Foley, E. Malone, P. Shearan, and L. Regan, "Determination of eleven coccidiostats in animal feed by liquid chromatography-tandem mass spectrometry at cross contamination levels," Analytica Chimica Acta, vol. 700, no. 12, pp. 26-33, 2011.

[26] R. Liu, W. Hei, P. He, and Z. Li, "Simultaneous determination of fifteen illegal dyes in animal feeds and poultry products by ultra-high performance liquid chromatography tandem mass spectrometry," Journal of Chromatography B, vol. 879, no. 24, pp. 2416-2422, 2011.

[27] W. Li, T. J. Herrman, and S. Y. Dai, "Determination of aflatoxins in animal feeds by liquid chromatography/tandem mass spectrometry with isotope dilution," Rapid Communications in Mass Spectrometry, vol. 25, no. 9, pp. 1222-1230, 2011.

[28] P. Delahaut, G. Pierret, N. Ralet, M. Dubois, and N. Gillard, "Multi-residue method for detecting coccidiostats at carryover level in feed by HPLC-MS/MS," Food Additives and Contaminants-Part A, vol. 27, no. 6, pp. 801-809, 2010.

[29] C. Van Poucke, F. Dumoulin, and C. Van Peteghem, "Detection of banned antibacterial growth promoters in animal feed by liquid chromatography-tandem mass spectrometry: optimisation of the extraction solvent by experimental design," Analytica Chimica Acta, vol. 529, no. 1-2, pp. 211-220, 2005.

[30] C. Van Poucke, K. De Keyser, A. Baltusnikiene, J. D. G. McEvoy, and C. Van Peteghem, "Liquid chromatographictandem mass spectrometric detection of banned antibacterial growth promoters in animal feed," Analytica Chimica Acta, vol. 483, no. 1-2, pp. 99-109, 2003.

[31] S. O'Connor, J. Locke, and D. S. Aga, "Addressing the challenges of tetracycline analysis in soil: extraction, clean-up, and matrix effects in LC-MS," Journal of Environmental Monitoring, vol. 9, no. 11, pp. 1254-1262, 2007.

[32] M. Lavén, T. Alsberg, Y. Yu, M. Adolfsson-Erici, and H. Sun, "Serial mixed-mode cation- and anion-exchange solid-phase 
extraction for separation of basic, neutral and acidic pharmaceuticals in wastewater and analysis by high-performance liquid chromatography-quadrupole time-of-flight mass spectrometry," Journal of Chromatography A, vol. 1216, no. 1, pp. 49-62, 2009.

[33] A. Tölgyesi, L. Tölgyesi, V. K. Sharma, M. Sohn, and J. Fekete, "Quantitative determination of corticosteroids in bovine milk using mixed-mode polymeric strong cation exchange solid-phase extraction and liquid chromatography-tandem mass spectrometry," Journal of Pharmaceutical and Biomedical Analysis, vol. 53, no. 4, pp. 919-928, 2010.

[34] D. R. Baker and B. Kasprzyk-Hordern, "Multi-residue analysis of drugs of abuse in wastewater and surface water by solid-phase extraction and liquid chromatography-positive electrospray ionisation tandem mass spectrometry," Journal of Chromatography A, vol. 1218, no. 12, pp. 1620-1631, 2011. 


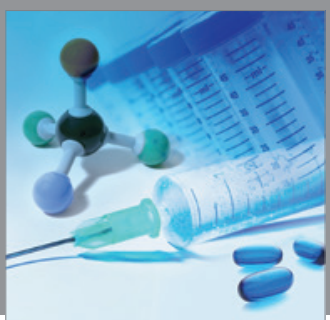

International Journal of

Medicinal Chemistry

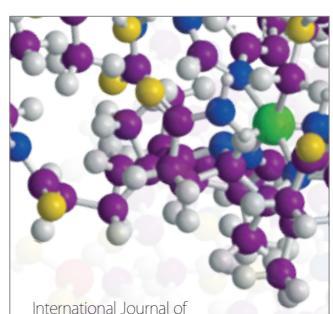

Carbohydrate Chemistry

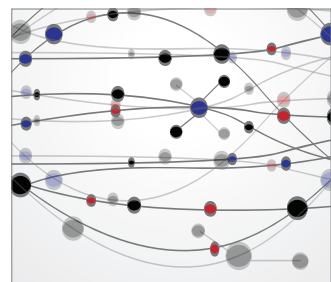

The Scientific World Journal
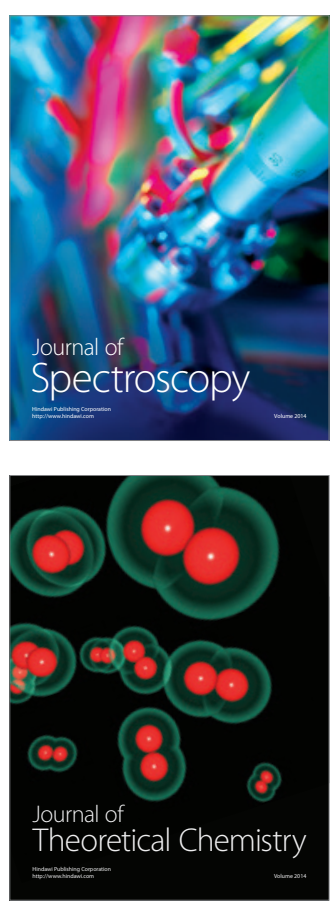
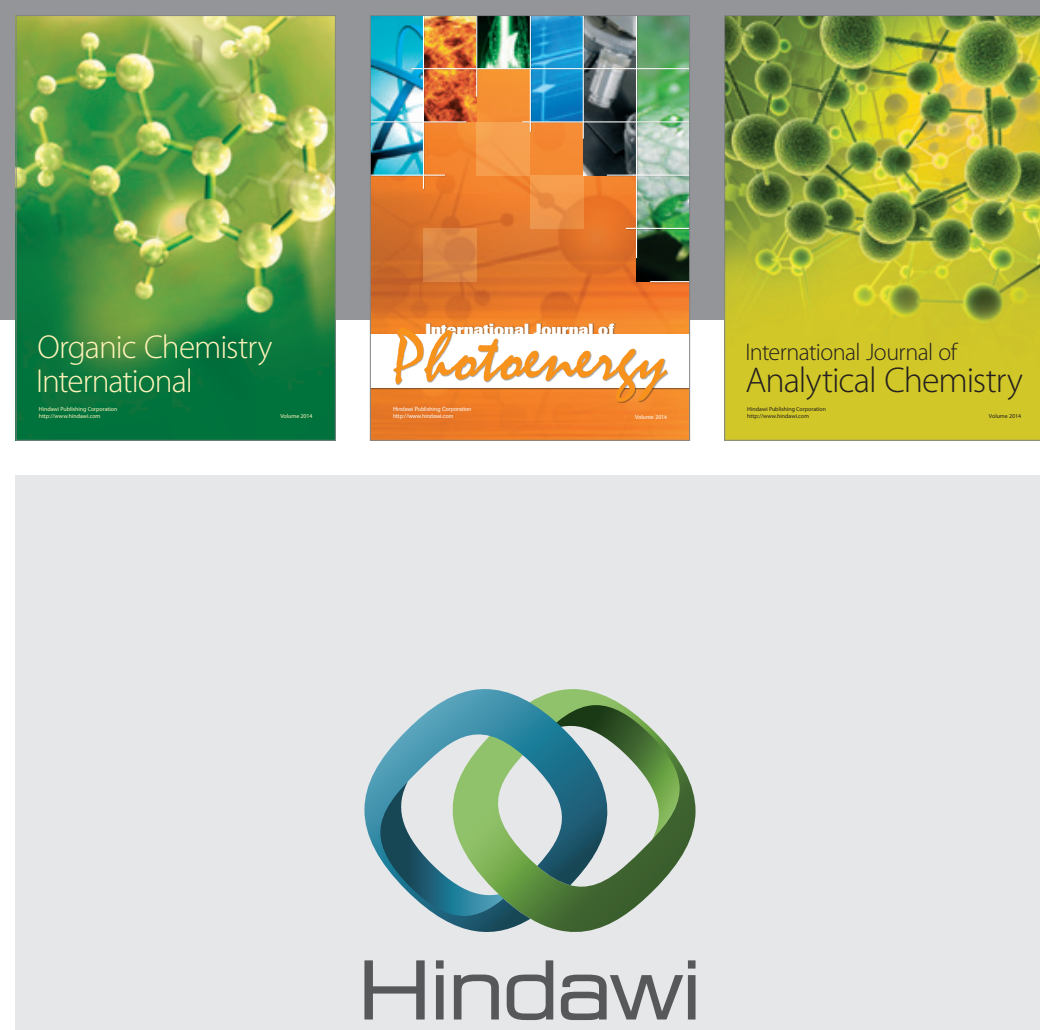

Submit your manuscripts at

http://www.hindawi.com
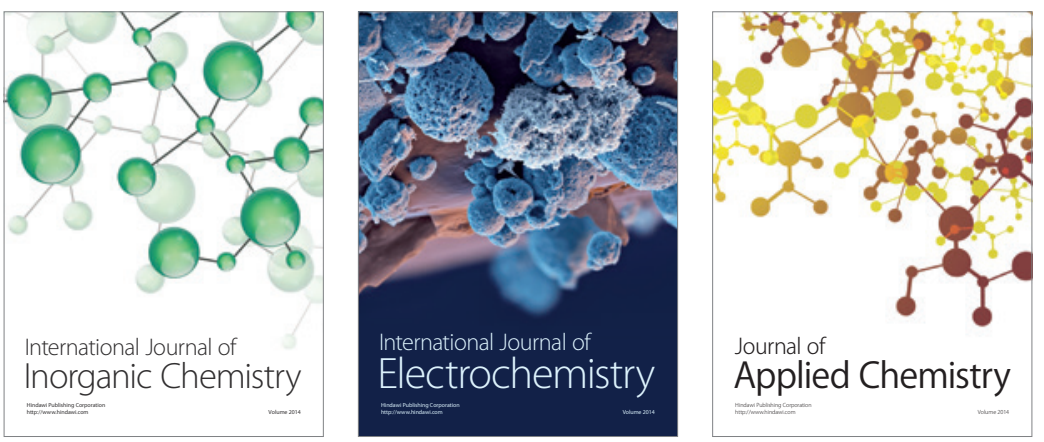

Journal of

Applied Chemistry
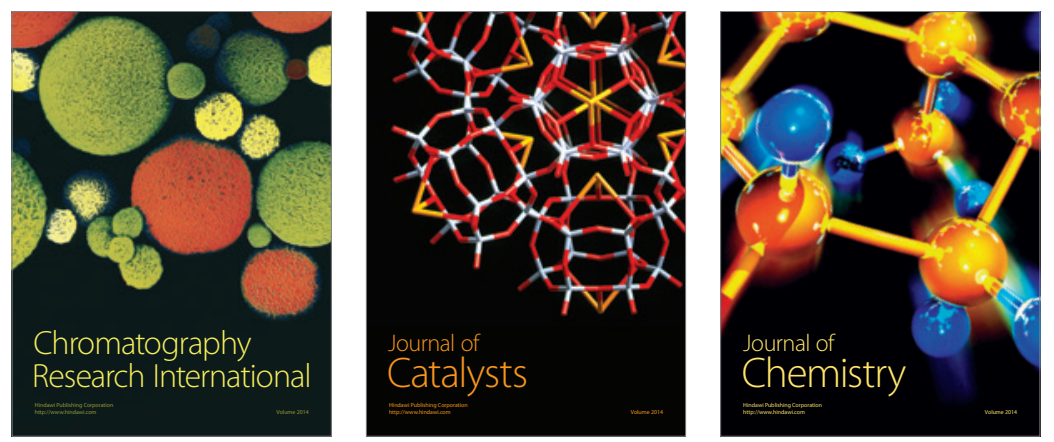
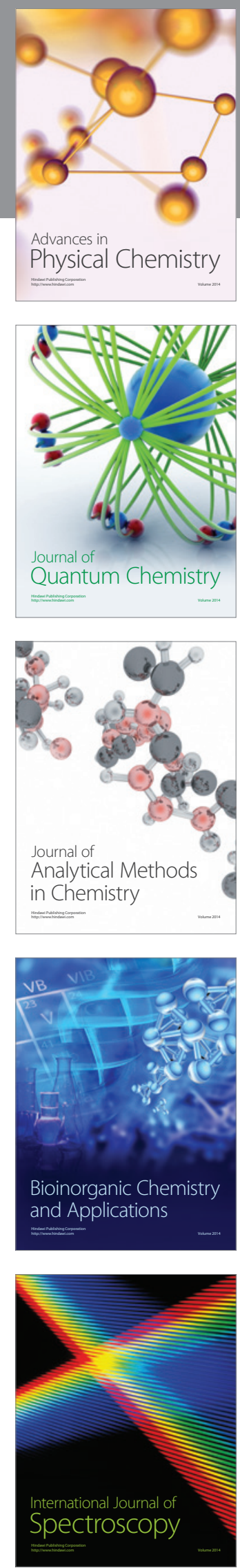